\title{
DESIGN AND SYNTHESIS OF NOVEL IMIDAZO[1,2-A]PYRIDINE DERIVATIVES AND THEIR ANTI-BACTERIAL ACTIVITY
}

\section{PUSHPALATHA BUDUMURU*, SRINIVASARAO GOLAGANI, VENKATA SIVA SATYANARAYANA KANTAMREDDI}

Department of Chemistry, Institute of Science, Gandhi Institute of Technology and Management Deemed To Be University, Visakhapatnam, Andhra Pradesh, India. Email: pushpalatha.budumuru@gmail.com

Received: 26 March 2018, Revised and Accepted: 01 May 2018

\section{ABSTRACT}

Objective: The present study aims to synthesize a novel derivatives of Imidazo[1,2-a]pyridines and the compounds were evaluated for their antibacterial activity.

Methods: A series of newly synthesized compounds were characterized by ${ }^{1} \mathrm{H}$-nuclear magnetic resonance (NMR), ${ }^{13} \mathrm{C}-\mathrm{NMR}$, Fourier transform infrared, mass spectral analysis, and screened for their antibacterial activity by disc diffusion method. Molecular docking studies were performed with a bacterial beta subunit of DNA gyrase using Auto Dock 4.2.6, and the docked conformations were analyzed using visual molecular dynamics.

Results: The structural activity relationship of the synthesized imidazo[1,2-a]pyridine derivatives was studied against Gram-positive and Gramnegative bacteria. Among the synthesized compounds N-benzyl-4-((2-(6-methyl-2-(p-tolyl)imidazo[1,2-a]pyridin-3-yl) acetamido)methyl) benzamide (9a) are possessing high activity against Bacillus subtilis. The zone of inhibition produced by the compound 9 a is wider than that of remaining compounds used in this study.

Conclusion: The synthesized compounds exhibited good antibacterial activity in comparison with standard drug streptomycin. This suggests that the compound 9a and its analogs are exerting their activity by probably inhibiting bacterial beta subunit of DNA gyrase.

Keywords: Imidazo[1,2-a]pyridine, Antibacterial activity, Disc diffusion method, Docking.

(c) 2018 The Authors. Published by Innovare Academic Sciences Pvt Ltd. This is an open access article under the CC BY license (http://creativecommons. org/licenses/by/4. 0/) DOI: http://dx.doi.org/10.22159/ajpcr.2018.v11i8.26241

\section{INTRODUCTION}

The emergence of human pathogenic microorganisms which is resistant to major classes of antibiotics has increased in recent years, due to the indiscriminate use of antimicrobial drugs [1]. This has caused many clinical problems in the treatment of infectious diseases, and the antibiotics that are commonly used are sometimes associated with adverse effects such as hypersensitivity, allergic reactions, and immunosuppression in the host [2]. Thus, the discovery of new antimicrobial agents is an urgent need. The situation seems to be worse in developing countries where many people with life-threatening infections may have no access to effective antimicrobials due to economic constrains [3]. The wide attention is therefore drawn on imidazopyridine derivatives due to their diverse biological activities and clinical applications such as antiviral [4], antiulcer [5], antibacterial [6,7] antifungal [8,9] antiprotozoal [10,11] antiherpes [12], and anti-inflammatory [13,14]. Several pyridines that are isolated from natural sources possess a broad spectrum of therapeutic activity. In the designing of new drugs, the development of hybrid molecules through the combination of different pharmacophores may lead to the formation of different compounds with interesting biological and pharmacological profiles.

\section{METHODS}

All the laboratory chemicals were supplied by Merck, Loba Chime Pvt. Ltd., and Spectrochem Pvt. Ltd., India. The purity of the compounds was checked by analytical thin layer chromatography (TLC) using Merck60GF254 aluminum coated TLC plates. Chloroform:methanol (90:10) was used as a solvent system. TLC spots were visualized with ultraviolet light/iodine vapors. The Fourier transform infrared (FT-IR) spectra were recorded on Shimadzu - FTIR 8300 spectrometer using $1 \% \mathrm{KBr}$ pellets. Mass spectra were recorded on SHIMADZU QP 500 spectrometer. All the ${ }^{1} \mathrm{H}$ and ${ }^{13} \mathrm{C}$-nuclear magnetic resonance (NMR) spectra were scanned on Bruker $300 \mathrm{MHz}$ spectrometer using $\mathrm{CDCl}_{3}$ or dimethyl sulfoxide (DMSO)- $\mathrm{d}_{6}$ as a solvent. Chemical shifts are expressed in $\delta$-scale (ppm) relative to tetramethylsilane as an internal standard.

\section{Chemistry}

Title compounds were synthesized from 4-methyl acetophenone as starting compound, which was depicted in the scheme 1.

\section{6-Methyl-2-p-tolyl-imidazo[1,2-a]pyridine (3)}

To the cooled solution of 4-methyl acetophenone $(1.5 \mathrm{~g}, 0.0074 \mathrm{~mol})$ in methanol, aluminum chloride $(0.492 \mathrm{~g}, 0.0037 \mathrm{~mol})$ was added under stirring at $0-5^{\circ} \mathrm{C}$. To this solution bromine $(0.592 \mathrm{~g}, 0.0074 \mathrm{~mol})$ was added slowly and stirred for $30 \mathrm{~min}$. To this reaction mixture, aqueous sodium carbonate and 2-amino 5-methyl pyridine solution $(0.7992 \mathrm{~g}$, $0.0074 \mathrm{~mol}$ ) were added at $25-35^{\circ} \mathrm{C}$. The completion of the reaction was monitored by TLC. The reaction mixture was poured into ice-cold water and the resulting solid was crystallized with ethanol and dried under vacuum to yield the cream color solid of 3 (1.26 g, yield: $54.8 \%$, mass spectrometry [MS]: $m / z 223.1(\mathrm{M}+1)$.

\section{$N, N$-dimethyl-1-(6-methyl-2-(p-tolyl)imidazo[1,2-a]pyridin-3-yl)} methenamine (4)

In a round bottom flask, the compound $3(1.0 \mathrm{~g}, 0.0045 \mathrm{~mol})$ was dissolved in acetic acid. To this solution, aqueous dimethylamine solution $(0.243 \mathrm{ml}, 0.0054 \mathrm{~mol})$ and formalin solution $(20 \mathrm{ml})$ were added, and the reaction mixture was subjected to reflux for $3 \mathrm{~h}$. The completion of the reaction was monitored by TLC. The reaction mixture was poured into crushed ice and basified using aqueous sodium hydroxide solution $(20 \mathrm{ml})$. The resulting precipitate was filtered, washed with water, dried, and crystallized from ethanol to give pale yellow solid of 4 (0.74 g, yield: 59.60\%). MS: m/z $280.2(\mathrm{M}+1)$. 
2-(6-methyl-2-(p-tolyl)imidazo[1,2-a]pyridin-3-yl) acetonitrile (5)

In a round bottom flask, the compound $4(1.0 \mathrm{~g}, 0.0035 \mathrm{~mol})$ was dissolved in $10 \mathrm{ml}$ of acetone. To this solution, precooled methyl iodide $(0.5 \mathrm{~g}, 0.0035 \mathrm{~mol})$ was added, and the reaction mixture was stirred at RT for $3 \mathrm{~h}$. The resulting solid was added to sodium cyanide $(0.176 \mathrm{~g}$, $0.0035 \mathrm{~mol}$ ) solution and heated for $2 \mathrm{~h}$. The reaction mixture was poured into ice-cold water and acidified with glacial acetic acid. The solid mass was filtered, dried and recrystallized using methanol to yield white solid of 5 (0.62 g, yield: 68.8\%) MS: $m / z 262.1(M+1)$.

(6-Methyl-2-p-tolyl-imidazo[1, 2-a]pyridin-3-yl)-acetic acid (6) In a round bottom flask, compound $5(1.5 \mathrm{~g}, 0.0057 \mathrm{~mol})$ was dissolved in methanol $(20 \mathrm{~mL})$. To this solution, potassium hydroxide $(0.161 \mathrm{~g}$, $0.0028 \mathrm{~mol})$ was added as an aqueous solution $(10 \mathrm{~mL})$. The reaction mixture was stirred under heating for $2 \mathrm{~h}$. The solvents were evaporated under reduced pressure. The obtained crude product was dissolved in ice-cold water and neutralized with Con. HCl. The resulting solid was filtered and dried in vacuum. 0.98 g, Yield: 71\%. MS: m/z $281.1(\mathrm{M}+1)$.

Ethyl 4-((2-(6-methyl-2-(p-tolyl)imidazo[1,2-a]pyridin-3-yl) acetamido)methyl)benzoate (7)

In a round bottom flask, the compound $6(1.0 \mathrm{~g}, 0.0036 \mathrm{~mol})$ was dissolved in dichloromethane $(25 \mathrm{~mL})$. To this solution, TBTU $(2.44 \mathrm{~g}$, $0.0072 \mathrm{~mol})$ and Triethylamine $(1.09 \mathrm{~mL}, 0.0108 \mathrm{~mol})$ were added. Then ethyl 4-(aminomethyl)benzoate $(0.773 \mathrm{~g}, 0.0043 \mathrm{~mol})$ was added and heated under reflux for $3 \mathrm{~h}$. The completion of the reaction was monitored by TLC, and after evaporation of the solvent, the reaction mixture was extracted with ethyl acetate. The organic layer was washed with sodium bicarbonate solution, water, and brine solution, which was separated and dried over anhydrous sodium sulfate. The evaporation of solvent yielded target compound ( 0.82 g, yield: $48.2 \%$ ) MS: m/z 441.1 $(\mathrm{M}+1)$.

4-((2-(6-Methyl-2-(p-tolyl)imidazo[1,2-a]pyridin-3-yl) acetamido)methyl)benzoic acid (8)

The compound $7(1.0 \mathrm{~g}, 0.0023 \mathrm{~mol})$ was dissolved in methanol $(30 \mathrm{~mL})$. To this solution, $10 \mathrm{ml}$ of sodium hydroxide solution was added. The reaction mixture was stirred for $1 \mathrm{hr}$ at room temperature. After completion of the reaction, the solvents were evaporated under reduced pressure. The crude product was dissolved in water and washed with ethyl acetate solution. The organic layer was discarded. The water layer having the product of sodium salt was neutralized with Con. $\mathrm{HCl}$. The obtained precipitate is filtered and dried in vacuum. $0.64 \mathrm{~g}$ Yield: 67.36\%. MS: $\mathrm{m} / \mathrm{z} 414.2(\mathrm{M}+1)$.

\section{General method for synthesis of compounds (9a-1)}

In a round bottom flask, the compound $8(0.2 \mathrm{~g}, 0.00048 \mathrm{~mol})$ was dissolved in $20 \mathrm{ml}$ of dichloromethane. To this, TBTU (0.32 g, $0.00096 \mathrm{~mol})$ and diisopropylamine $(0.145 \mathrm{ml}, 0.0014 \mathrm{~mol})$ were added and stirred for 5 min under nitrogen atmosphere. Then, amine $(0.00058 \mathrm{~mol})$ was added and the reaction was refluxed for $3 \mathrm{~h}$. The completion of the reaction was monitored by TLC. The reaction mixture was extracted with ethyl acetate after completion of the reaction. The organic layer was washed with sodium bicarbonate solution, water, and brine solution. Then, it was separated and dried over anhydrous sodium sulfate. The evaporation of solvent and appropriate recrystallization was yielded the target compounds (9a-l).

N-benzyl-4-((2-(6-methyl-2-(p-tolyl)imidazo[1,2-a]pyridin-3-yl) acetamido)methyl) benzamide (9a)

Yield 31.5\%; ${ }^{1} \mathrm{H}-\mathrm{NMR}$ (400 MHz, DMSO-d6) ppm: 2.30 (s, 3H, $\mathrm{CH}_{3}$ ); 2.35 (s, $\left.3 \mathrm{H}, \mathrm{CH}_{3}\right) ; 4.04\left(\mathrm{~d}, 2 \mathrm{H}, \mathrm{CH}_{2}\right) ; 4.40-4.41\left(\mathrm{~d}, 2 \mathrm{H}, \mathrm{CH}_{2}\right) ; 4.49-4.50(\mathrm{~d}$, $\left.2 \mathrm{H}, \mathrm{CH}_{2}\right) ; 7.12-7.14(\mathrm{~m}, 1 \mathrm{H}, \mathrm{Ar}-\mathrm{H}) ; 7.24-7.26(\mathrm{~m}, 3 \mathrm{H}, \mathrm{Ar}-\mathrm{H}) ; 7.32$ (m, 4H, Ar-H); 7.36-7.38 (m, 2H, Ar-H); 7.48-7.51 (d, 1H, Ar-H); 7.68-7.70 (d, 2H, Ar-H); 7.87-7.89 (d, 2H, Ar-H); 8.18 (s, 1H, Ar-H); 8.89 (s, 1H,
NH); 9.03 (s, $1 \mathrm{H}, \mathrm{NH}) .{ }^{13} \mathrm{C}-\mathrm{NMR}(400 \mathrm{MHz}, \mathrm{DMSO}-d 6)$ ppm: $\delta 169.06$, 166.39 (C of N-amide), 143.39, 143.26, 140.19, 137.06,133.34,132.27, (C of 1-benzene, 1-C-C, 1-C=C), 129.52,128.75, 128.19, 127.78, 127.62, 127.50, 127.19, (CH of 1-benzene), 122.63,121.34, 116.46, 115.16, (CH of 1-ethylene), 43.03,42.64, 31.32,21.19,18.18 (CH3 of -C=C,-C=N,-C,$\mathrm{C}(=\mathrm{O})-\mathrm{N},-\mathrm{N})$. FT-IR $\left(\mathrm{KBr}, \mathrm{cm}^{-1}\right): 3450(\mathrm{~N}-\mathrm{H}) ; 3080(\mathrm{Ar}-\mathrm{H}), 2929(\mathrm{C}-\mathrm{H})$, 1634 (C=0), 1553 (C=N), 1439 (C=C), 1392 (C-N). MS: m/z 503.1 (M+1).

N-(2-chlorobenzyl)-4-((2-(6-methyl-2-(p-tolyl)imidazo[1,2-a] pyridin-3-yl)acetamido)methyl benzamide (9b)

Yield 22.7\%; ${ }^{1} \mathrm{H}-\mathrm{NMR}\left(400 \mathrm{MHz}, \mathrm{CDCl}_{3}\right.$ ) ppm: 2.31 (s, 3H, $\mathrm{CH}_{3}$ ); 2.34 (s, $\left.3 \mathrm{H}, \mathrm{CH}_{3}\right) ; 4.04\left(\mathrm{~d}, 2 \mathrm{H}, \mathrm{CH}_{2}\right) ; 4.43\left(\mathrm{~d}, 2 \mathrm{H}, \mathrm{CH}_{2}\right) ; 4.70-4.71\left(\mathrm{~d}, 2 \mathrm{H}, \mathrm{CH}_{2}\right)$; 7.17-7.19 (m, 1H, Ar-H); 7.22 (m, 1H, Ar-H); 7.23-7.24 (m, 2H, Ar-H); 7.37 (m, 4H, Ar-H); 7.36-7.38 (m, 2H, Ar-H); 7.43-7.45 (d, 1H, Ar-H); 7.55-7.57 (d, 2H, Ar-H); 7.61-7.66 (d, 2H, Ar-H); 7.97 (s, 1H, Ar-H); 8.19 (s, 1H, NH); $8.63(\mathrm{~s}, 1 \mathrm{H}, \mathrm{NH})$. FT-IR $\left(\mathrm{KBr}, \mathrm{cm}^{-1}\right): 3437(\mathrm{~N}-\mathrm{H}) ; 3014(\mathrm{Ar}-$ H), $2920(\mathrm{C}-\mathrm{H}), 1670(\mathrm{C}=\mathrm{O}), 1543(\mathrm{C}=\mathrm{N}), 1441(\mathrm{C}=\mathrm{C}), 1334$ (C-N). MS: $\mathrm{m} / \mathrm{z} 538.1(\mathrm{M}+1)$.

$N$-(4-fluorobenzyl)-4-((2-(6-methyl-2-(p-tolyl)imidazo[1,2-a] pyridin-3-yl)acetamido)methyl) benzamide (9c)

Yield 24.6\%; ${ }^{1} \mathrm{H}-\mathrm{NMR}$ (400 MHz, DMSO-d6) ppm: 2.31 (s, 3H, $\mathrm{CH}_{3}$ ); 2.35 (s, $\left.3 \mathrm{H}, \mathrm{CH}_{3}\right) ; 4.04\left(\mathrm{~d}, 2 \mathrm{H}, \mathrm{CH}_{2}\right) ; 4.38-4.39\left(\mathrm{~d}, 2 \mathrm{H}, \mathrm{CH}_{2}\right) ; 4.44-4.46(\mathrm{~d}, 2 \mathrm{H}$, $\left.\mathrm{CH}_{2}\right) ; 7.12-7.19(\mathrm{~m}, 3 \mathrm{H}, \mathrm{Ar}-\mathrm{H}) ; 7.24-7.26(\mathrm{~d}, 2 \mathrm{H}, \mathrm{Ar}-\mathrm{H}) ; 7.32-7.36(\mathrm{~m}$, $4 \mathrm{H}, \mathrm{Ar}-\mathrm{H}$ ); 7.50-7.53 (d, 1H, Ar-H); 7.65-7.67 (d, 2H, Ar-H); 7.83-7.85 (d, $2 \mathrm{H}, \mathrm{Ar}-\mathrm{H}) ; 8.21(\mathrm{~s}, 1 \mathrm{H}, \mathrm{Ar}-\mathrm{H}) ; 8.85-8.88(\mathrm{t}, 1 \mathrm{H}, \mathrm{NH}) ; 9.00-9.03(\mathrm{t}$, 1H, NH). FT-IR (KBr, cm ${ }^{-1}$ ): 3427 (N-H); 3019 (Ar-H), 2922 (C-H), 1667 $(\mathrm{C}=0), 1545(\mathrm{C}=\mathrm{N}), 1423$ (C=C), $1330(\mathrm{C}-\mathrm{N}) \mathrm{MS}: \mathrm{m} / \mathrm{z} 521.2(\mathrm{M}+1)$.

$N$-(3,5-bis(trifluoromethyl)benzyl)-4-((2-(6-methyl-2-(p-tolyl) imidazo[1,2-a]pyridin-3-yl) acetamido)methyl)benzamide (9d)

Yield 22.5\%; ${ }^{1} \mathrm{H}-\mathrm{NMR}$ (400 MHz, DMSO-d6) ppm: 2.28 (s, 3H, $\mathrm{CH}_{3}$ ); 2.33 (s, 3H, $\left.\mathrm{CH}_{3}\right) ; 4.03\left(\mathrm{~d}, 2 \mathrm{H}, \mathrm{CH}_{2}\right) ; 4.38-4.39\left(\mathrm{~d}, 2 \mathrm{H}, \mathrm{CH}_{2}\right) ; 4.64-4.65(\mathrm{~d}, 2 \mathrm{H}$, $\mathrm{CH}_{2}$ ); 7.11-7.13 (m, 1H, Ar-H); 7.22-7.25 (m, 2H, Ar-H); 7.36-7.38 (d, 2H, Ar-H); 7.48-7.50 (d, 1H, Ar-H); 7.65-7.67 (d, 2H, Ar-H); 7.84-7.89 (m, 2H, Ar-H); 8.01 (s, 3H, Ar-H); 8.17 (s, 1H, Ar-H); 8.85-8.88 (t, 1H, $\mathrm{NH})$; $9.16-9.19(\mathrm{t}, 1 \mathrm{H}, \mathrm{NH})$. FT-IR $\left(\mathrm{KBr}, \mathrm{cm}^{-1}\right): 3441(\mathrm{~N}-\mathrm{H}) ; 3081(\mathrm{Ar}-\mathrm{H})$, 2923 (C-H), 1638 (C=0), 1544 (C=N), 1421 (C=C), 1381 (C-N). MS: m/z $639.2(M+1)$.

4-((2-(6-methyl-2-(p-tolyl)imidazo[1,2-a]pyridin-3-yl) acetamido)methyl)- $N$-(4-methyl benzyl)benzamide (9e)

Yield 38.5\%; ${ }^{1} \mathrm{H}-\mathrm{NMR}$ (400 MHz, DMSO-d6) ppm: 2.26 (s, 3H, $\mathrm{CH}_{3}$ ); 2.34 (s, 3H, $\left.\mathrm{CH}_{3}\right) ; 2.36$ (s, 3H, $\left.\mathrm{CH}_{3}\right) ; 4.07$ (d, $\left.2 \mathrm{H}, \mathrm{CH}_{2}\right) ; 4.38-4.39$ (d, $2 \mathrm{H}, \mathrm{CH}_{2}$ ); 4.42-4.43 (d, $2 \mathrm{H}, \mathrm{CH}_{2}$ ); 7.11-7.20 (m, 4H, Ar-H); 7.28-7.30 (d, 2H, ArH); 7.34-7.36 (d, 3H, Ar-H); 7.60-7.65 (m, 3H, Ar-H); 7.84-7.86 (d, 2H, Ar-H); 8.35 (s, 1H, Ar-H); 8.88-8.91 (t, 1H, NH); 8.95-8.98 (t, 1H, NH). FT-IR $\left(\mathrm{KBr}, \mathrm{cm}^{-1}\right): 3425(\mathrm{~N}-\mathrm{H}) ; 3012(\mathrm{Ar}-\mathrm{H}), 2934(\mathrm{C}-\mathrm{H}), 1667(\mathrm{C}=\mathrm{O})$, $1532(\mathrm{C}=\mathrm{N}), 1426$ (C=C), 1349 (C-N). MS: m/z $517.3(\mathrm{M}+1)$.

$N$-(3-methoxybenzyl)-4-((2-(6-methyl-2-(p-tolyl)imidazo[1,2-a] pyridin-3-yl)acetamido) methyl)benzamide (9f)

Yield 19.9\%; ${ }^{1} \mathrm{H}-\mathrm{NMR}$ (400 MHz, DMSO-d6) ppm: 2.30 (s, 3H, $\mathrm{CH}_{3}$ ); 2.35 (s, 3H, $\left.\mathrm{CH}_{3}\right) ; 3.37$ (s, 3H, OCH $)$; $4.03\left(\mathrm{~d}, 2 \mathrm{H}, \mathrm{CH}_{2}\right) ; 4.39-4.40\left(\mathrm{~d}, 2 \mathrm{H}, \mathrm{CH}_{2}\right)$; 4.42-4.43 (d, $\left.2 \mathrm{H}, \mathrm{CH}_{2}\right) ; 7.11-7.14(\mathrm{~d}, 1 \mathrm{H}, \mathrm{Ar}-\mathrm{H}) ; 7.23-7.25(\mathrm{~d}, 1 \mathrm{H}, \mathrm{Ar}-\mathrm{H})$; 7.36-7.43 (m, 4H, Ar-H); 7.47-7.55 (m, 2H, Ar-H); 7.65-7.72 (m, 3H, ArH); 7.87-7.89 (d, 2H, Ar-H); 7.97-7.99 (d, 1H, Ar-H); 8.17 (s, 1H, Ar-H); 8.85-8.88 (t, 1H, NH); 8.96-8.97 (t, 1H, NH). FT-IR $\left(\mathrm{KBr}, \mathrm{cm}^{-1}\right): 3427$ $(\mathrm{N}-\mathrm{H}) ; 3023(\mathrm{Ar}-\mathrm{H}), 2922(\mathrm{C}-\mathrm{H}), 1672(\mathrm{C}=\mathrm{O}), 1552(\mathrm{C}=\mathrm{N}), 1443(\mathrm{C}=\mathrm{C})$, 1339 (C-N). MS: m/z 533.1 (M+1).

$N$-(4-methoxybenzyl)-4-((2-(6-methyl-2-(p-tolyl)imidazo[1,2-a] pyridin-3-yl)acetamido) methyl)benzamide (9g)

Yield 24.6\%; ${ }^{1} \mathrm{H}-\mathrm{NMR}$ (400 MHz, DMSO-d6) ppm: 2.35 (s, 3H, $\mathrm{CH}_{3}$ ); 2.36 (s, $\left.3 \mathrm{H}, \mathrm{CH}_{3}\right) ; 3.72\left(\mathrm{~s}, 3 \mathrm{H}, \mathrm{OCH}_{3}\right) ; 4.07\left(\mathrm{~d}, 2 \mathrm{H}, \mathrm{CH}_{2}\right) ; 4.37-4.38\left(\mathrm{~d}, 2 \mathrm{H}, \mathrm{CH}_{2}\right)$; 4.39-4.40 (d, 2H, $\mathrm{CH}_{2}$ ); 6.87-6.89 (d, 2H, Ar-H); 7.22-7.24 (d, 2H, Ar-H); 
7.29-7.35 (m, 4H, Ar-H); 7.60-7.64 (m, 3H, Ar-H); 7.83-7.85 (m, 2H, $\mathrm{Ar}-\mathrm{H}) ; 8.36$ (s, 1H, Ar-H); 8.87-8.90 (t, 1H, NH); 8.92-8.96 (t, 1H, NH). FT-IR $\left(\mathrm{KBr}, \mathrm{cm}^{-1}\right): 3427(\mathrm{~N}-\mathrm{H}) ; 3023(\mathrm{Ar}-\mathrm{H}), 2922(\mathrm{C}-\mathrm{H}), 1672(\mathrm{C}=\mathrm{O})$, $1552(\mathrm{C}=\mathrm{N}), 1443$ (C=C), 1339 (C-N). MS: m/z $533.2(\mathrm{M}+1)$.

\section{4-((2-(6-methyl-2-(p-tolyl)imidazo[1,2-a]pyridin-3-yl) acetamido)methyl)- $N$-(pyridin-4-ylmethyl)benzamide (9h)}

Yield 32.3\%; ${ }^{1} \mathrm{H}-\mathrm{NMR}$ (400 MHz, DMSO-d6) ppm: 2.30 (s, 3H, $\mathrm{CH}_{3}$ ); 2.35 (s, $\left.3 \mathrm{H}, \mathrm{CH}_{3}\right) ; 4.03\left(\mathrm{~d}, 2 \mathrm{H}, \mathrm{CH}_{2}\right) ; 4.38-4.40\left(\mathrm{~d}, 2 \mathrm{H}, \mathrm{CH}_{2}\right) ; 4.48-4.50(\mathrm{~d}, 2 \mathrm{H}$, $\left.\mathrm{CH}_{2}\right)$; 7.11-7.14 (m, 1H, Ar-H); 7.23-7.30 (m, 4H, Ar-H); 7.36-7.38 (d, $2 \mathrm{H}, \mathrm{Ar}-\mathrm{H}$ ); 7.47-7.50 (d, $1 \mathrm{H}, \mathrm{Ar}-\mathrm{H}) ; 7.66-7.68$ (d, 2H, Ar-H); 7.85-7.87 (d, 2H, Ar-H); 8.17 (s, 1H, Ar-H); 8.49-8.50 (m, 2H, Ar-H); 8.85-8.88 (t, 1H, NH); 9.07-9.10 (t, 1H, NH). FT-IR (KBr, cm $\left.{ }^{-1}\right): 3459$ (N-H); 3024 (Ar-H), $2934(\mathrm{C}-\mathrm{H}), 1636$ (C=0), 1559 (C=N), 1427 (C=C), 1346 (C-N). MS: $\mathrm{m} / \mathrm{z} 504.2(\mathrm{M}+1)$.

$N$-(4-chlorobenzyl)-4-((2-(6-methyl-2-(p-tolyl)imidazo[1,2-a] pyridin-3-yl)acetamido) methyl)benzamide (9i)

Yield 39.3\%; ${ }^{1} \mathrm{H}-\mathrm{NMR}$ (400 MHz, $\mathrm{CDCl}_{3}$ ) ppm: 2.33 (s, 3H, $\mathrm{CH}_{3}$ ); 2.38 (s, $\left.3 \mathrm{H}, \mathrm{CH}_{3}\right) ; 4.05$ (d, $\left.2 \mathrm{H}, \mathrm{CH}_{2}\right) ; 4.42-4.43\left(\mathrm{~d}, 2 \mathrm{H}, \mathrm{CH}_{2}\right) ; 4.58-4.60(\mathrm{~d}, 2 \mathrm{H}$, $\left.\mathrm{CH}_{2}\right)$; 6.13-6.15 (t, $\left.1 \mathrm{H}, \mathrm{NH}\right)$; 6.37-6.40 (t, $\left.1 \mathrm{H}, \mathrm{NH}\right) ; 7.08-7.11(\mathrm{~m}, 1 \mathrm{H}$, $\mathrm{Ar}-\mathrm{H})$; 7.14-7.16 (d, 2H, Ar-H); 7.21-7.23 (d, 2H, Ar-H); 7.27-7.32 (m, 4H, Ar-H); 7.49-7.51 (d, 1H, Ar-H); 7.56-7.58 (d, 2H, Ar-H); 7.64-7.66 (d, 2H, Ar-H); 7.75 (s, 1H, Ar-H). FT-IR $\left(\mathrm{KBr}, \mathrm{cm}^{-1}\right): 3456(\mathrm{~N}-\mathrm{H}) ; 3012$ $(\mathrm{Ar}-\mathrm{H}), 2914$ (C-H), 1635 (C=0), 1558 (C=N), 1427 (C=C), 1323 (C-N). MS: $\mathrm{m} / \mathrm{z} 537.2(\mathrm{M}+1)$.

4-((2-(6-methyl-2-(p-tolyl)imidazo[1,2-a]pyridin-3-yl) acetamido)methyl)- $N$-(4-(trifluoro methyl)benzyl)benzamide (9j)

Yield 22.6\%; ${ }^{1} \mathrm{H}-\mathrm{NMR}$ (400 MHz, $\mathrm{CDCl}_{3}$ ) ppm: 2.35 (s, 3H, $\mathrm{CH}_{3}$ ); 2.37 (s, $\left.3 \mathrm{H}, \mathrm{CH}_{3}\right) ; 4.04\left(\mathrm{~d}, 2 \mathrm{H}, \mathrm{CH}_{2}\right) ; 4.41-4.43\left(\mathrm{~d}, 2 \mathrm{H}, \mathrm{CH}_{2}\right) ; 4.69-4.70(\mathrm{~d}, 2 \mathrm{H}$, $\left.\mathrm{CH}_{2}\right)$; 6.15-6.18 (t, $\left.1 \mathrm{H}, \mathrm{NH}\right) ; 6.56-6.58(\mathrm{t}, 1 \mathrm{H}, \mathrm{NH}) ; 7.14-7.21(\mathrm{~m}, 5 \mathrm{H}$, Ar-H); 7.45-7.47 (d, 2H, Ar-H); 7.51-7.53 (m, 2H, Ar-H); 7.59-7.61 (m, 3H, Ar-H); 7.67-7.69 (d, 2H, Ar-H); 7.83 (s, 1H, Ar-H). FT-IR (KBr, cm ${ }^{-1}$ ): 3457 (N-H); 3028 (Ar-H), 2936 (C-H), 1639 (C=O), 1549 (C=N), 1435 $(\mathrm{C}=\mathrm{C}), 1327$ (C-N). MS: m/z $571.2(\mathrm{M}+1)$.

4-((2-(6-methyl-2-(p-tolyl)imidazo[1,2-a]pyridin-3-yl) acetamido)methyl)- $N$-(pyridin-3-yl methyl)benzamide (9k)

Yield 35.8\%; ${ }^{1} \mathrm{H}$-NMR ( $400 \mathrm{MHz}$, DMSO-d6) ppm: 2.29 (s, 3H, $\mathrm{CH}_{3}$ ); 2.34 (s, 3H, $\left.\mathrm{CH}_{3}\right) ; 4.03$ (d, $\left.2 \mathrm{H}, \mathrm{CH}_{2}\right) ; 4.38-4.39$ (d, $\left.2 \mathrm{H}, \mathrm{CH}_{2}\right) ; 4.48-4.50(\mathrm{~d}, 2 \mathrm{H}$, $\left.\mathrm{CH}_{2}\right)$; 7.12-7.14 (d, $\left.1 \mathrm{H}, \mathrm{Ar}-\mathrm{H}\right) ; 7.22-7.24(\mathrm{~d}, 2 \mathrm{H}, \mathrm{Ar}-\mathrm{H}) ; 7.33-7.37$ (m, $3 \mathrm{H}, \mathrm{Ar}-\mathrm{H}) ; 7.48-7.50$ (d, 1H, Ar-H); 7.65-7.71 (m, 3H, Ar-H); 7.83-7.85 (d, 2H, Ar-H); 8.17 (s, 1H, Ar-H); 8.44-8.46 (m, 1H, Ar-H); 8.54-8.55 (d, 1H, Ar-H); 8.85-8.88 (t, 1H, NH); 9.05-9.08 (t, 1H, NH). FT-IR (KBr, $\left.\mathrm{cm}^{-1}\right)$ : $3440(\mathrm{~N}-\mathrm{H}) ; 3012(\mathrm{Ar}-\mathrm{H}), 2918(\mathrm{C}-\mathrm{H}), 1667(\mathrm{C}=\mathrm{O}), 1536(\mathrm{C}=\mathrm{N})$, 1451 (C=C), 1348 (C-N). MS: m/z $504.2(\mathrm{M}+1)$.

$N$-(3,4-dichlorobenzyl)-4-((2-(6-methyl-2-(p-tolyl) imidazo[1,2-a]pyridin-3-yl)acetamido) methyl)benzamide (9l)

Yield 26.7\%; ${ }^{1} \mathrm{H}-\mathrm{NMR}$ (400 MHz, DMSO-d6) ppm: 2.30 (s, 3H, $\mathrm{CH}_{3}$ ); 2.35 (s, 3H, $\left.\mathrm{CH}_{3}\right) ; 4.03\left(\mathrm{~d}, 2 \mathrm{H}, \mathrm{CH}_{2}\right)$; 4.39-4.40 (d, $\left.2 \mathrm{H}, \mathrm{CH}_{2}\right) ; 4.56-4.58(\mathrm{~d}, 2 \mathrm{H}$, $\left.\mathrm{CH}_{2}\right) ; 7.12-7.14(\mathrm{~m}, 1 \mathrm{H}, \mathrm{Ar}-\mathrm{H}) ; 7.23-7.25(\mathrm{~d}, 2 \mathrm{H}, \mathrm{Ar}-\mathrm{H}) ; 7.36-7.43(\mathrm{~d}$, $3 \mathrm{H}, \mathrm{Ar}-\mathrm{H}$ ); 7.48-7.55 (m, 2H, Ar-H); 7.65-7.72 (d, 2H, Ar-H); 7.87-7.89 (d, 2H, Ar-H); 7.96-7.99 (d, 1H, Ar-H); 8.17 (s, 1H, Ar-H); 8.85-8.88 (t, $1 \mathrm{H}, \mathrm{NH})$; 8.96-8.99 (t, 1H, NH). FT-IR $\left(\mathrm{KBr}, \mathrm{cm}^{-1}\right): 3455(\mathrm{~N}-\mathrm{H}) ; 3016(\mathrm{Ar}-$ H), $2916(\mathrm{C}-\mathrm{H}), 1674(\mathrm{C}=\mathrm{O}), 1558(\mathrm{C}=\mathrm{N}), 1412(\mathrm{C}=\mathrm{C}), 1235$ (C-N). MS: $\mathrm{m} / \mathrm{z} 571.2(\mathrm{M}+1)$.

\section{Antibacterial activity}

The antibacterial activities of newly synthesized compounds (9a-l) were carried out by disc diffusion method [15] against four microorganisms, namely Escherichia coli (ATCC-25922), Staphylococcus aureus (ATCC9144), Klebsiella pneumoniae (ATCC-13883), and Bacillus subtilis (ATCC-6051). In this method, 24-h-old Mueller-Hinton broth cultures of test bacteria were adjusted to standard subculture dilution and swabbed uniformly on solidified sterile Mueller-Hinton agar plates. The plates were allowed to dry for $15 \mathrm{~min}$ and then used for the sensitivity test.

Experiments were carried out at two different concentrations (500 $\mu \mathrm{g}$ and $1000 \mu \mathrm{g}$ ) of the test samples. DMSO was used as diluents to get desired concentration of test sample. Each concentration was injected on pre-sterilized disc papers (Whatman No. 1, diameter $6 \mathrm{~mm}$ ) and then they were placed over Mueller-Hinton agar surface. Each test plate comprises six discs including one positive control and one negative control, and they were placed about equidistance to each other. Standard streptomycin drug $(10 \mu \mathrm{g})$ was used as a positive control, and DMSO (100\%) was used as a negative control. Then, the inoculated plates were incubated at $37^{\circ} \mathrm{C}$ for $24 \mathrm{~h}$, and the zone of inhibition was recorded and expressed in millimeters as its antibacterial activity.

\section{Molecular docking studies \\ Hardware components}

In the present work, all the docking calculations and computational analysis were carried out with the Hi-end server (Pentium 3.4 MHzs, AMD Athlon 64 bit, Qudra-core processor with 8 GB RAM) manufactured by HCL Corporation, India.

Molecular docking is a key tool to predict the predominant binding mode(s) of a ligand with a target protein of known threedimensional (3D) structure. As a preprocessing step, ChemDraw Ultra 12.0 was used to generate 3D structure files in PDB format from two-dimensional drawn structures of ligand molecules. Docking calculations were performed using AutoDock 4.2.6. AutoDock is a suite of automated docking tools designed to predict how small molecules, such as substrates or drug candidates, bind to a receptor of known 3D structure. The 3D structures were optimized using the PRODRG server. The PDB formats of the protein macromolecule and small molecules are converted to Autodock's PDBQT format. From the AutoDock Vina, the macromolecule was added with gasteiger partial charges, and further polar hydrogens were added. AutoDock performs the docking of the ligand to a set of grids describing the target protein; AutoGrid pre-calculates these grids. In addition to using them for docking, the atomic affinity grids can be visualized. The docking of a small molecule to the macromolecule was focused on the active site of the beta subunit of bacterial DNA Gyrase. Six residues spanning the active site were treated as flexible. The total number of rotatable bonds for the ligands and flexible residues were calculated. Grid Box of dimension 60, 60, and 60 in XYZ dimensions was placed around the active site of the protein structure with the center of the grid box set to $2.614,-3.136$, and 20.702 in $\mathrm{X}, \mathrm{Y}$, and Z dimensions, respectively. The binding free energies between receptor and the ligands were obtained in terms of $\mathrm{Kcal} / \mathrm{mol}$. The docked conformations were visualized analyzed using visual molecular dynamics.

\section{RESULTS AND DISCUSSIONS}

\section{Chemistry}

The target molecules (9a-l) were synthesized from 4-methyl acetophenone 1 as showed in Scheme 1 . The proposed compounds were confirmed and characterized individually by their respective FTIR, NMR, and Mass spectral data. Physicochemical properties of synthesized compounds were represented in Table 1. The reaction of precursor 1 with 2-amino 5 -methyl pyridine in the presence of bromine and aluminum chloride provided 6-Methyl-2-p-tolyl-imidazo[1,2-a] pyridine [16]. The mass spectrum of 3 displayed a protonated molecular ion at $m / z$ 223. In the course of synthesis, the compound 3 reacts with acetic acid, dimethylamine and formalin at $90^{\circ} \mathrm{C}$ for $3 \mathrm{~h}$ to yield dimethyl aminomethyl derivative of 4 and the structure was confirmed by mass spectra at $\mathrm{m} / \mathrm{z} 280$. The related compound 5 formed during reaction with methyl iodide to form quaternary ammonium salt and further reacted with sodium cyanide yielding cyano derivative. 


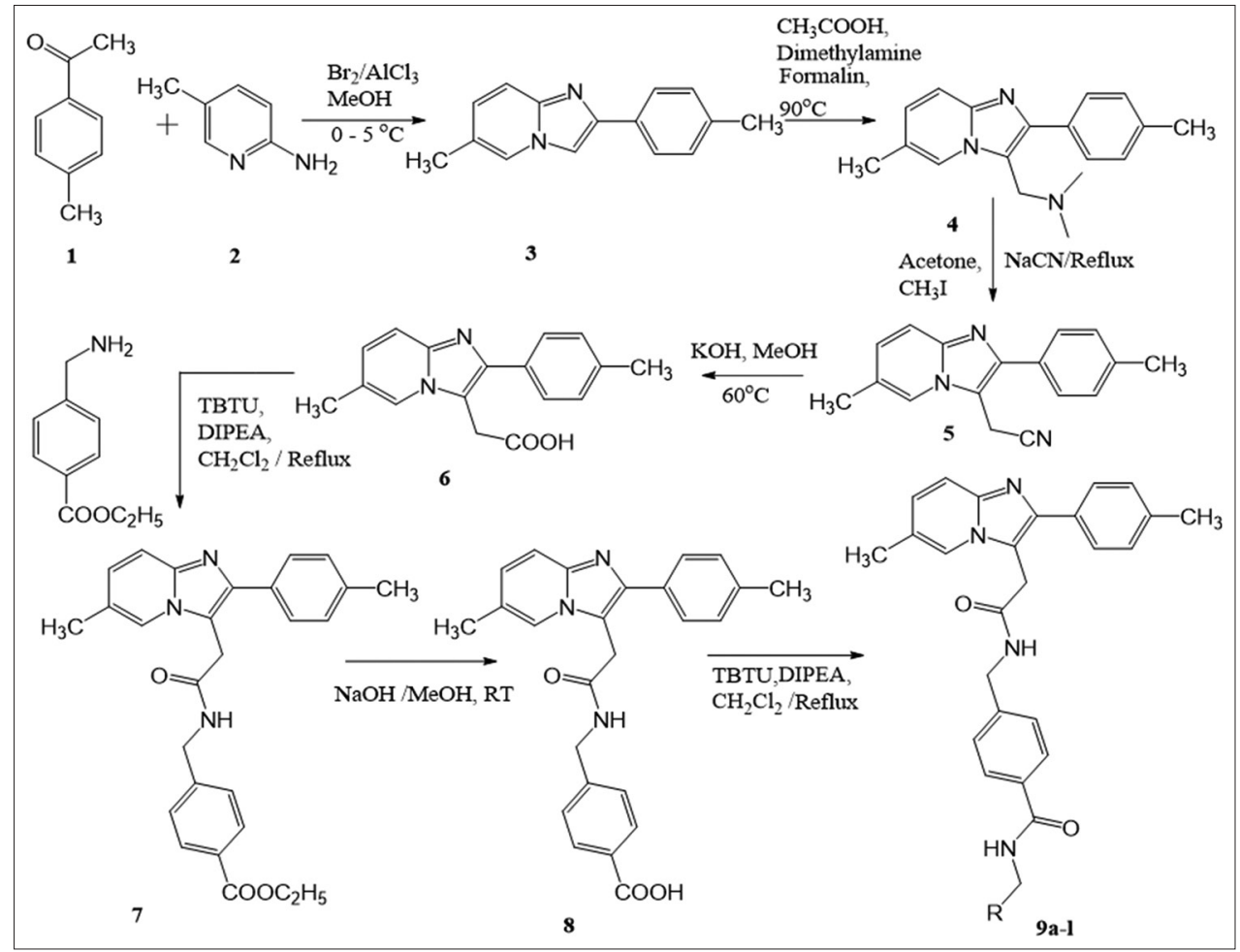

Scheme 1: Synthetic route for compound 9a-1

Table 1: Physicochemical properties of synthesized compounds

\begin{tabular}{|c|c|c|c|c|}
\hline Compound & $\mathbf{R}$ & Molecular formula & Molecular weight & Yield (\%) \\
\hline $9 a$ & & $\mathrm{C}_{32} \mathrm{H}_{30} \mathrm{~N}_{4} \mathrm{O}_{2}$ & 502.6 & 31.5 \\
\hline $9 b$ & & $\mathrm{C}_{32} \mathrm{H}_{29} \mathrm{ClN}_{4} \mathrm{O}_{2}$ & 537.05 & 22.7 \\
\hline $9 c$ & & $\mathrm{C}_{32} \mathrm{H}_{29} \mathrm{FN}_{4} \mathrm{O}_{2}$ & 520.59 & 24.6 \\
\hline $9 \mathrm{~d}$ & & $\mathrm{C}_{34} \mathrm{H}_{28} \mathrm{~F}_{6} \mathrm{~N}_{4} \mathrm{O}_{2}$ & 638.6 & 22.5 \\
\hline $9 e$ & & $\mathrm{C}_{33} \mathrm{H}_{32} \mathrm{~N}_{4} \mathrm{O}_{2}$ & 516.63 & 38.5 \\
\hline
\end{tabular}

(Contd...) 
Table 1: (Continued)

\begin{tabular}{|c|c|c|c|c|}
\hline Compound & $\mathbf{R}$ & Molecular formula & Molecular weight & Yield (\%) \\
\hline $9 f$ & & $\mathrm{C}_{32} \mathrm{H}_{30} \mathrm{~N}_{4} \mathrm{O}_{3}$ & 532.63 & 19.9 \\
\hline $9 g$ & & $\mathrm{C}_{32} \mathrm{H}_{30} \mathrm{~N}_{4} \mathrm{O}_{3}$ & 532.63 & 24.6 \\
\hline $9 \mathrm{~h}$ & & $\mathrm{C}_{31} \mathrm{H}_{29} \mathrm{~N}_{5} \mathrm{O}_{2}$ & 503.59 & 32.3 \\
\hline $9 \mathrm{i}$ & & $\mathrm{C}_{32} \mathrm{H}_{29} \mathrm{ClN}_{4} \mathrm{O}_{2}$ & 537.05 & 39.3 \\
\hline $9 j$ & & $\mathrm{C}_{32} \mathrm{H}_{29} \mathrm{~F}_{3} \mathrm{~N}_{4} \mathrm{O}_{2}$ & 570.6 & 22.6 \\
\hline $9 \mathrm{k}$ & & $\mathrm{C}_{31} \mathrm{H}_{29} \mathrm{~N}_{5} \mathrm{O}_{2}$ & 503.59 & 35.8 \\
\hline 91 & & $\mathrm{C}_{32} \mathrm{H}_{28} \mathrm{Cl}_{2} \mathrm{~N}_{4} \mathrm{O}_{2}$ & 571.49 & 26.7 \\
\hline
\end{tabular}

This compound was further hydrolyzed using potassium hydroxide and methanol to yield the acid derivative of compound 6. Compound 7 was quantitatively synthesized using compound 6 which reacts with TBTU, Ethyl 4-(aminomethyl)benzoate in the presence of triethylamine under reflux for $3 \mathrm{~h}$. The high resolution-MS spectrum of 7 displayed a protonated molecular ion at $\mathrm{m} / \mathrm{z} 442$. Compound 7 was hydrolyzed using aqueous sodium hydroxide in methanol to form the corresponding acid derivative 8 . The mass spectrum of 8 showed a molecular ion at $\mathrm{m} / \mathrm{z}$ 414. The final compound of imidazo[1, 2-a] pyridine derivatives $9 \mathrm{a}-\mathrm{l}$ was prepared by amide coupling with TBTU, triethylamine with corresponding benzylamines. The mass spectrum of the compound 9a showed a molecular ion peak at m/z 503 and a sharp band at $1634 \mathrm{~cm}^{-1}$ was observed in the IR spectrum which is recognized as amide $\mathrm{C}=0$ stretching. The FT-IR results contributed some additional information for functional groups such as $\mathrm{NH}$ and $\mathrm{CH}$ groups. In the ${ }^{1} \mathrm{H}-\mathrm{NMR}$ spectrum, the two singlet signals at $2.30 \mathrm{ppm}$ and $2.35 \mathrm{ppm}$ were observed and they were characterized for methyl groups. The amide $\mathrm{NH}$ groups were observed at $8.89 \mathrm{ppm}$ and $9.03 \mathrm{ppm}$. Similarly, three $\mathrm{CH}_{2}$ units were appeared at 4.04 (d), 4.41(d), and 4.49 (d). The aromatic protons also present at their corresponding regions.

\section{Biology}

Antibacterial activity of Imidazo[1,2-a]pyridine derivatives

The multidrug resistance both in the community and hospitals has been the major concerns to public health and scientific community worldwide $[17,18]$. The development of antimicrobial agents to treat infectious diseases has been one of the most notable achievements of the past century. The increased use of antimicrobial agents that are available in the market has resulted in the development of resistance to commonly used drugs with important implications for morbidity, mortality, and health-care costs $[19,20]$. All the synthesized imidazo[1,2-a]pyridine compoundswere screened for their antibacterial activity by disc diffusion method. The compounds were screened for their in vitro antimicrobial activity against E. coli (ATCC-25922), S. aureus (ATCC-9144), K. pneumoniae (ATCC-13883), and B. subtilis (ATCC6051) and were compared with standard drug Streptomycin and the zones of inhibition were calculated. All the synthesized compounds at the concentration of $1000 \mu \mathrm{g}$ showed promising inhibition against the tested microbial pathogens. Among the synthesized compounds, the compound 9a, 9c, 9e, and 9g containing benzyl, 4-fluorobenzyl, 4-methylbenzyl, and 4-methoxybenzyl substituents demonstrated 
Table 2: Antibacterial activity of Imidazo[1,2-a] pyridine derivatives

\begin{tabular}{|c|c|c|c|c|c|c|c|c|}
\hline \multicolumn{9}{|c|}{ Zone of inhibition (mm) } \\
\hline \multirow[t]{2}{*}{ Compound code } & \multicolumn{2}{|c|}{ S. aureus } & \multicolumn{2}{|c|}{ B. subtilis } & \multicolumn{2}{|l|}{ E. coli } & \multicolumn{2}{|c|}{ K. pneumoniae } \\
\hline & $500 \mu \mathrm{g}$ & $1000 \mu \mathrm{g}$ & $500 \mu \mathrm{g}$ & $1000 \mu \mathrm{g}$ & $500 \mu \mathbf{g}$ & $1000 \mu \mathrm{g}$ & $\mathbf{5 0 0} \mu \mathbf{g}$ & $1000 \mu \mathrm{g}$ \\
\hline $9 a$ & 7 & 10 & 12 & 15 & 11 & 13 & - & 12 \\
\hline $9 b$ & - & 8 & 9 & 13 & 9 & 12 & - & 10 \\
\hline $9 c$ & - & 8 & 10 & 12 & 10 & 14 & 8 & 10 \\
\hline $9 d$ & - & 7 & 10 & 11 & 8 & 11 & - & 8 \\
\hline $9 \mathrm{e}$ & 7 & 9 & 10 & 14 & 11 & 14 & 7 & 11 \\
\hline $9 \mathrm{f}$ & - & 9 & 10 & 13 & 10 & 13 & 7 & 10 \\
\hline $9 \mathrm{~g}$ & - & 9 & 9 & 14 & 9 & 13 & - & 11 \\
\hline $9 \mathrm{~h}$ & - & 9 & 9 & 12 & 10 & 12 & - & 10 \\
\hline $9 \mathrm{i}$ & - & 8 & 9 & 13 & 10 & 13 & - & 9 \\
\hline $9 j$ & - & 10 & 9 & 12 & 10 & 12 & 8 & 11 \\
\hline $9 \mathrm{k}$ & - & 9 & 9 & 13 & 9 & 12 & 7 & 9 \\
\hline 91 & - & 9 & 10 & 11 & 9 & 13 & - & 8 \\
\hline Streptomycin & 22 & & 23 & & 23 & & 22 & \\
\hline
\end{tabular}

S. aureus: Staphylococcus aureus, B. subtilis: Bacillus subtilis, E. coli: Escherichia coli, K. pneumoniae: Klebsiella pneumoniae

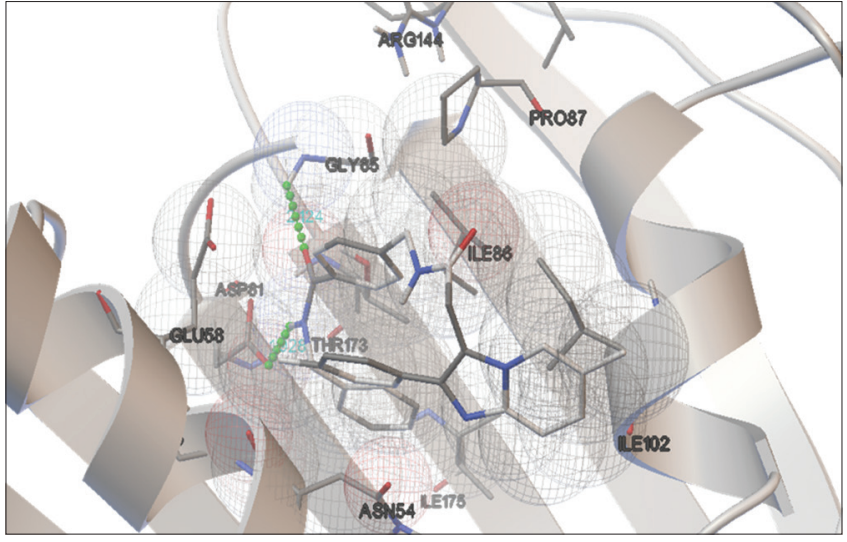

Fig. 1: 9a bound to the ATPase active site of bacterial DNA Gyrase (from Bacillus subtilis) showed an inhibition constant of $60 \mu \mathrm{M}$. The estimated binding free energy of this interaction is $6 \mathrm{Kcal} / \mathrm{mol}$. The hydrogen bond formed between $9 \mathrm{a}$ and side chain carboxyl group of Asp 61 and the hydrogen bond between 9a and backbone $\mathrm{NH}$ of Gly65 is shown as green spheres

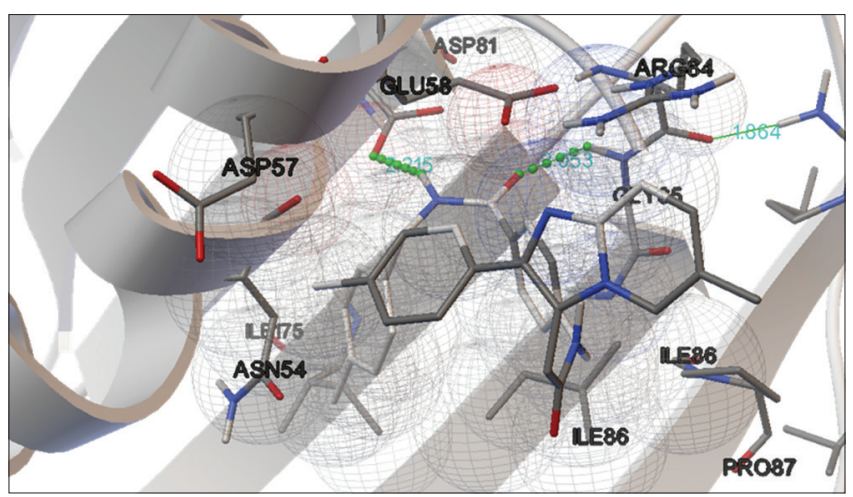

Fig. 2: 9e bound to the ATPase active site of bacterial DNA Gyrase (from Bacillus subtilis) showed an inhibition constant of $1 \mathrm{mM}$.

The estimated binding free energy of this interaction is $5 \mathrm{Kcal} / \mathrm{mol}$. The hydrogen bond formed between $9 \mathrm{e}$ and side chain carboxyl group of Glu 58 and the hydrogen bond between $9 e$ and backbone $\mathrm{NH}$ of Gly85 is shown as green spheres

inhibition against all the pathogens at the concentration of $1000 \mu \mathrm{g}$. The compound 9c and 9e having 4-fluorobenzyl, 4-methylbenzyl substituents had shown moderate activity against E.coli organism.

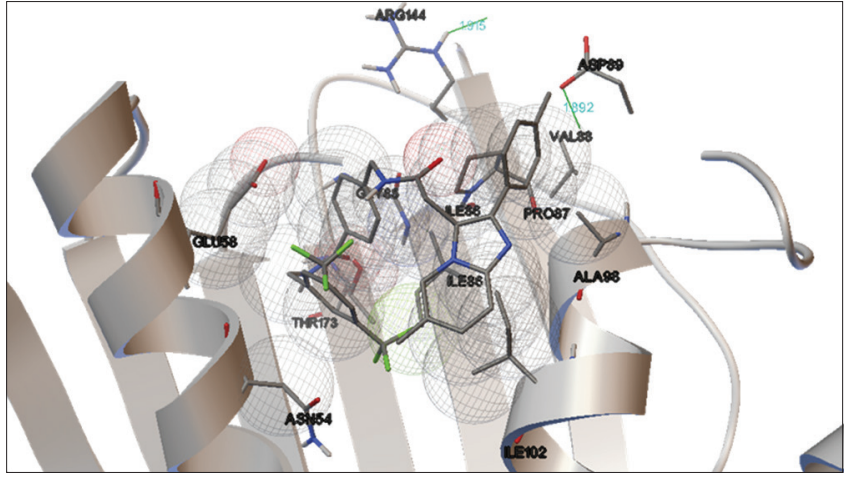

Fig. 3: 9d bound to the ATPase active site of bacterial DNA Gyrase (from Bacillus subtilis) showed the weakest binding among all the analogs with a predicted inhibition constant of $29.8 \mathrm{mM}$. The estimated binding free energy of this interaction is $\sim-2 \mathrm{Kcal} / \mathrm{mol}$ No hydrogen bonds were formed between $9 \mathrm{~d}$ and protein. The interaction was found to be predominantly hydrophobic in nature

Furthermore, the compound 9e, 9g, and 9j containing 4-methylbenzyl, 4-methoxybenzyl, and 3,4,5-triflouromethyl benzyl substituents showed inhibition against $K$. pneumonia. All the synthesized compounds had showed less activity against $S$. aureus. Similarly, no activity was recorded by the compounds $9 \mathrm{a}, 9 \mathrm{~b}, 9 \mathrm{~d}, 9 \mathrm{~g}, 9 \mathrm{~h}, 9 \mathrm{i}$, and $9 \mathrm{l}$ at their lowest concentrations $(500 \mu \mathrm{g})$ tested against K. pneumoniae. Highest zone of inhibition was demonstrated by benzyl substituted compound 9a against $B$. subtilis formed a zone of inhibition of $15 \mathrm{~mm}$. The findings showed the antibacterial potential of the synthesized compounds against the standard antibiotic. The zone of inhibitions (in $\mathrm{mm}$ ) of the microorganisms is represented in Table 2.

The docking results are in conformity with the experimental observations (Figs. 1-3).

Compound 9a was found to have better binding score than other structural analogs used in this study. The zones of inhibition produced by this compound $9 \mathrm{a}$ are wider than that of remaining compounds used in this study. This suggests the fact that $9 \mathrm{a}$ and its analogs are exerting their activity by probably inhibiting bacterial beta subunit of DNA gyrase.

\section{CONCLUSION}

The synthesis of novel imidazo[1,2-a]pyridine derivatives was prepared by the conventional method. Nearly 12 compounds, namely 9a-l were synthesized successfully and confirmed by mass, FT-IR, and 
NMR spectrums. All the synthesized compounds were evaluated for their antibacterial activity. The results showed the good antibacterial potential of the synthesized compounds against the standard antibiotic at their highest concentration tested. These compounds may be further examined and used as pharmacologically active antibacterial compounds against common bacterial pathogens in the near future.

\section{ACKNOWLEDGMENTS}

The authors are very thankful to Sapala organics, Hyderabad, for providing NMR and Mass spectral data of synthesized compounds and to Greensmed Labs, Chennai, for providing facilities to carry out antibacterial activity.

\section{AUTHORS' CONTRIBUTIONS}

All authors have made considerable contributions to the work reported in the manuscript.

\section{CONFLICTS OF INTEREST}

The authors confirm that this article content has no conflicts of interest.

\section{REFERENCES}

1. Karaman I, Sahin F, Güllüce M. Antimicrobial activity of aqueous and methanol extracts of Juniperus oxycedrus L. J Ethnopharmacol 2003;85:231-5.

2. Mukherjee PK, Saritha GS, Suresh B. Antimicrobial potential of two different Hypericum species available in India. Phytother Res 2002;16:692-5.

3. Puerstinger G, Paeshuyse J, Declercq E, Neyts J. Antiviral 2, 5-disubstituted imidazo[4,5-c]pyridines from anti-pestivirus to antihepatitis C virus activity. Bioorg Med Chem Lett 2007;17:390-3.

4. Katsura Y, Nishino S, Inowe Y, Tomoi M, Takasugi H. Studies on antiulcer drugs II synthesis and antiulcer activities of imidazo[1,2-a]pyridinyl2-alkylaminobenzoxazoles and 5,6,7,8-tetrahydroimidazo[1,2- $a$ ] pyridinyl derivatives. Chem Pharm Bull 1992;40:371-80.

5. Rewankar GR, Matthews JR, Robins RK. Synthesis and antimicrobial activity of certain imidazo[1,2-a]pyrimidines. J Med Chem 1975;18:1253-5.

6. Rival Y, Grassy G, Michel G. Synthesis and antibacterial activity of some imidazo[1,2- $a$ pyrimidine derivatives. Chem Pharm Bull 1992;40:1170-6.

7. Fisher MH, Lusi A. Imidazo $[1,2-a]$ pyridine anthelmintic and antifungal agents. J Med Chem 1972;15:982-5.

8. Rival Y, Grassy G, Taudou A, Ecalle R. Antifungal activity in vitro of some imidazo[1,2-alpyrimidine derivatives. Eur J Med Chem 1991;26:13-8

9. Biftu T, Feng D, Fisher M, Liang G, Qian X, Scribner A, et al. Synthesis and SAR studies of very potent imidazopyridine antiprotozoal agents. Bioorg Med Chem Lett 2006;16:2479-83.

10. Ismail MA, Arafa RK, Wenzler T, Brun R, Tanious FA, Wilson WD, et al. Synthesis and antiprotozoal activity of novel bis-benzamidinoimidazo $[1,2-a]$ pyridines and 5,6,7,8-tetrahydro-imidazo [1,2- $a]$ pyridines. Bioorg Med Chem 2008;16:683-91.

11. Gudmundsson KS, Johns BA. Imidazo $[1,2-a]$ pyridines with potent activity against herpesviruses. Bioorg Med Chem Lett 2007;17:2735-9.

12. Maruyama Y, Anami K, Terasawa M, Goto K, Imayoshi T, Kadabe Y, et al. Anti-inflammatory activity of an imidazopyridine derivative (miroprofen), Arzneimittel Forsch 1981;31:1111-5.

13. Abignente E, Carariis P, Fattorusso E, Mayol L. Research on heterocyclic compounds XXIII phenyl derivatives of fused imidazole systems. J Heteroc Chem 1989;26:1875-80.

14. Moraski GC, Markley LD, Hipskind PA, Boshoff H, Cho S, Franzblau SG, et al. Advent of Imidazo[1,2-a]pyridine-3-carboxamides with potent multi and extended drug resistant antituberculosis activity. ACS Med Chem Lett 2011;2:466-70.

15. Bhardwaja V, Malleshappa NN, Jalhan S, Patel HM. Synthesis, and antimicrobial evaluation of new pyridine imidazo [2,1b]-1,3,4thiadiazole derivatives. J Saudi Chem Soc 2016:20;S406-10.

16. Aanandhi MV, Bhattacherjee D, Kamalraj R. Synthesis, docking and biological activity of various substituted zolpidem based GABAA inhibitors endowed potent hypnotic and sedative activity. Med Chem 2014;2:1-8.

17. van Duin D, Paterson D. Multidrug resistant bacteria in the community: Trends and lessons learned. Infect Dis Clin North Am 2016;30:377-90.

18. Sanjay MW, Ghorpade MV, Shivali VG, Annapurna GS, Rashmi MK. A study of vancomycin resistanct Enterococci isolated from urinary tract infections. Int J Pharm Pharm Sci 2015;7:337-9.

19. Fair RJ, Tor Y. Antibiotics and bacterial resistance in the $21^{\text {st }}$ Century. Perspect Med Chem 2014;6:25-64.

20. Balakrishnan J, Appalasamy JR. Skin infection and the global challenges: A Review. Int J Pharm Pharm Sci 2016;8:1-3. 